\title{
A role for midcingulate cortex in the interruptive effects of pain anticipation on attention
}

\author{
Christopher A Brown*, Anthony KP Jones \\ Human Pain Research Group, Clinical Sciences Building, Hope Hospital, Salford, M6 8HD, United Kingdom
}

\section{A R T I C L E I N F O}

\section{Article history:}

Accepted 10 June 2008

Available online $\mathrm{xxxx}$

\section{Keywords:}

Cingulate

Cognitive

EEG

LORETA

Nociception

Physiology

\begin{abstract}
A B S T R A C T
Objective: To investigate the anticipatory neural processes associated with the interruptive effects of pain anticipation on attention.

Methods: Sustained attention was measured in healthy subjects $(n=24)$ by the number of task errors in a go/no-go task involving temporal discrimination of non-painful cutaneous electrical stimuli. Painful distractors were randomly delivered to the same spatial location and the resulting increases in task errors (indicating interruption of attention) were measured. In a separate task the same subjects attended to the spatial location of painful laser stimuli delivered to the right forearm, and we localized the sources of anticipatory ERPs prior to stimulation.

Results: Pain anticipation was associated with activation of pain matrix areas including bilateral insula, mid- and posterior cingulate cortices, and bilateral inferior parietal cortices. Subjects with greater pain-related increases in task errors found the pain to be more unpleasant, and showed increased early pain-related anticipatory processing in the midcingulate cortex. They also demonstrated reduced processing in a spatial attention network comprising posterior cingulate and inferior parietal cortices.

Conclusions: The results suggest a role for the midcingulate cortex in interrupting attention during pain anticipation.

Significance: Individuals with greater anticipatory midcingulate responses may be predisposed to developing chronic pain and hypervigilance toward clinical pain symptoms.

(c) 2008 International Federation of Clinical Neurophysiology. Published by Elsevier Ireland Ltd. All rights reserved.
\end{abstract}

\section{Introduction}

Attention is one of the best-studied psychological phenomena in relation to pain. In understanding interactions between attention and pain, it is helpful to make a distinction between automatic (involuntary) and intentional (voluntary) attentional processes. Automatic processes of attention are thought to underlie pain hypervigilance, which is significantly related to important clinical issues surrounding chronic pain, particularly psychogenic pain (Crombez et al., 2005). Pain hypervigilance can be defined as greater than usual automatic attentional selection of pain-related threats out of the field of sensory experience (Peters et al., 2000). Hypervigilant attention can occur during anticipation of future pain and is therefore thought to be driven by fear and anxiety (Nutt et al., 2002; Bogels and Mansell, 2004). Experimental studies have shown that the effects of pain-related fear in augmenting pain perception is mediated by pain hypervigilance (Arntz et al., 1994; Crombez et al., 1998; Crombez et al., 1998; Keogh et al., 2001). In patients with chronic pain, the level of pain-related fear predicts

\footnotetext{
* Corresponding author. Tel.: +44 1612064528.

E-mail address: christopher.brown@manchester.ac.uk (C.A Brown).
}

the extent to which their pain causes attentional interference (Crombez et al., 1998, 1999). Hence, the result of hypervigilance is that pain has a more interruptive effect on attention (Eccleston and Crombez, 1999; Crombez et al., 2005).

The neural correlates in the brain that mediate the interaction between attention and pain processing have recently been the subject of investigation (Seminowicz et al., 2004; Seminowicz and Davis, 2007). However, it is not clear which specific areas of the 'pain matrix' in the brain mediate the interaction between attention and pain processing. Neuroimaging studies commonly reveal neural responses to acute pain in the midcingulate cortex, which is thought to have both attentional and affective functions in relation to painful stimuli (Peyron et al., 2000; Garcia-Larrea et al., 2003; Vogt, 2005) and non-painful stimuli (Posner and Petersen, 1990; Bush et al., 2000). Studies investigating neural activity associated with sustained attention (using go/no-go tasks, stroop tasks) have implicated similar regions of the midcingulate cortex to those that occur in response to acute pain, although they do not exactly overlap (Davis et al., 1997; Derbyshire et al., 1998; Buffington et al., 2005). It has been suggested that midcingulate responses to pain may better reflect automatic orientation of attention toward pain that serves to interrupt attention to other 
stimuli or tasks (Peyron et al., 2000). By contrast, the intentional focusing of attention toward pain, and in particular its spatial location, has been linked to a right-lateralized attention network including inferior parietal cortex (Peyron et al., 1999; Kulkarni et al., 2005).

Neuroimaging studies using PET or fMRI are not well placed to study anticipatory processes that may be involved in mediating pain hypervigilance, owing to their poor temporal resolution. Anticipatory potentials preceding pain such as the Stimulus-Preceding Negativity (SPN) can be measured using EEG (Bocker et al., 1994; Brown et al., 2008). The SPN is not induced by the need for an immanent motor response, by contrast to the Contingent Negative Variation (CNV) and the Readiness Potential (RP) (see Brunia and van Boxtel, 2001 for a review). The SPN is thought to reflect anticipatory attention toward a stimulus, and is thought to be initiated by the relevant sensory cortices under some conditions (Brunia and van Boxtel, 2001).

In this study, we set out to measure the interruptive effect of pain anticipation on sustained attention and to determine anticipatory neural correlates of this effect. We hypothesized that subjects who show greater attentional interruption by anticipating pain would report greater pain unpleasantness at a given intensity. This was on the basis that greater attentional resources given to the anticipation of pain would result in greater pain unpleasantness. To test this, we designed a go/no-go task that measured the interruptive effect of pain anticipation on attention to the task. Subjects attended to the sensory aspects of electrical somatosensory stimuli, responding to all except infrequent oddball stimuli. Painful distractors (which subjects were told to ignore and were therefore task-irrelevant) were delivered during the task. Pain-related reductions in task performance as a result of the painful distractors were used to infer the extent to which attention was interrupted by pain anticipation.

Two aspects of the experimental design helped to ensure that the interruptive effects of the pain could be attributed to its anticipatory component. Firstly, target stimuli (which were used to measure attentional interruption) and subsequent non-target stimuli were never painful. Hence, pain-evoked responses were temporally differentiated from processing and responding to targets. Secondly, painful distractors were always delivered to the same spatial location as the task-relevant stimuli. Because pain is well known to automatically induce attentional orientation toward it, such a spatial coherence would be expected to increase task performance rather than interrupt it. Any interruptive effects of the pain could therefore be attributed to secondary affective processing (e.g. fear/anxiety) taking place during anticipation of pain, rather than shifts in spatial attention away from the task due to primary pain processing.

Our second hypothesis was that attentional interruption by pain anticipation would be associated with greater anticipatory responses to pain in the midcingulate cortex, in addition to anatomically connected regions known to form an attention network with this region (e.g. right inferior parietal cortex Baleydier and Mauguiere, 1980; Vogt and Pandya, 1987). In order to test this, we investigated the SPN during anticipation of pain in a separate task in which subjects focused on the spatial location of the pain to control for intentional processes of attention between the two tasks. By comparing data between the two tasks, anticipatory responses that correlated with the interruptive effects of pain were determined.

\section{Methods}

Twenty-four healthy right-handed subjects participated in the study (12 female, 12 male; mean age $34 \pm 14$ ). Subjects gave informed written consent, and the study was approved by Tameside and Glossop Local Research Ethics Committee.

\subsection{Experiment 1: Go/no-go task. Measuring the effect of pain anticipation on attention}

In order to measure the distractive effects of pain anticipation on sustained, task-relevant attention, we needed two conditions in which (1) attention was either affected by pain or not affected by pain, (2) the task was equally difficult in both painful and non-painful conditions apart from differences in difficulty resulting from differential painfulness, (3) pain-related changes in task performance could be attributed to pain anticipation rather than physiological factors such as the properties of peripheral nerves (such as their density, which may vary across individuals).

To achieve this, we designed a go/no-go task based on temporal discrimination in which subjects were required to respond to 'single' electrical pulses and withhold their response to 'double' pulses (Fig. 1). Electrical pulses were applied to the subjects' right index finger by passing a current between two electrodes placed $3 \mathrm{~cm}$ apart using a purpose-built constant current electrical stimulator. Initially, a psychophysics procedure determined individually tailored stimulus intensities for each subject. Applying single pulses of $200 \mu$ s duration, a ramping procedure was performed three times in order to determine the stimulus current required to induce three subjective intensity levels: 'low' intensity (non-painful, at twice the sensory threshold), 'medium' intensity (non-painful, at three-quarters of the pain threshold) and 'high' intensity (moderately painful, halfway between pain threshold and pain tolerance). In addition, double pulses were used during the study at the 'low' intensity, consisting of $2 \times 200 \mu$ s duration pulses with an interstimulus interval of $100 \mathrm{~ms}$.

Prior to commencing the experiment, subjects performed a sensory discrimination task in order to confirm that they were able to accurately discriminate single and double pulses. Subjects responded verbally to 20 low-intensity stimuli, consisting of a randomized sequence of single and double pulses, by indicating which type of pulse they perceived. Subjects that misperceived at least one of the pulses performed the task again at incrementally higher stimulus intensities until they scored $100 \%$ correct. This cor-

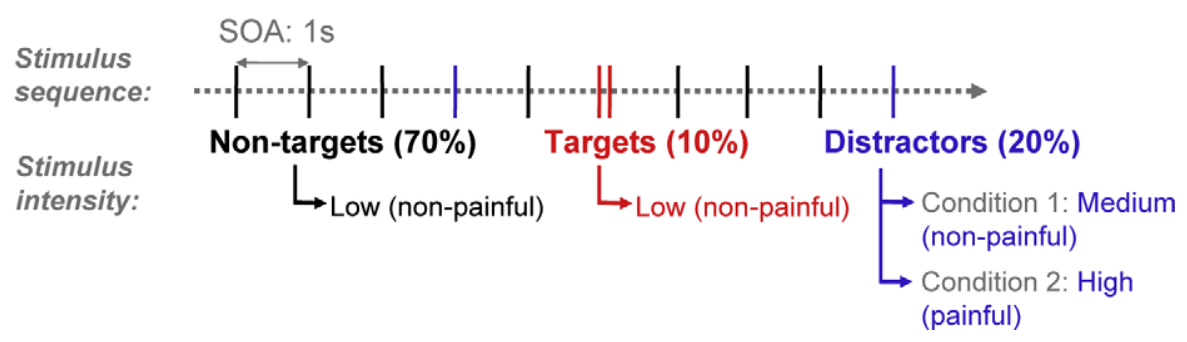

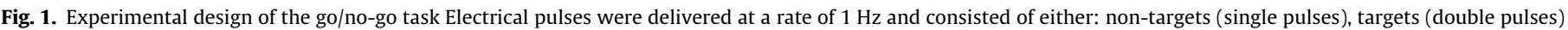

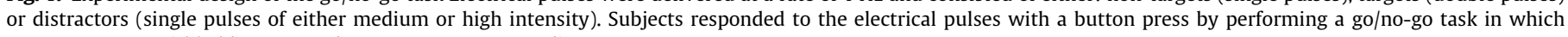
responses were withheld to targets but not non-targets or distractors. 
rected intensity was used for the 'low' intensity pulses during the experiment. Despite these corrections, 'low' intensity pulses were found to be less than half the intensity of the 'medium' pulses for all subjects (see Fig. 2).

During the main part of the experiment, subjects performed a go/no-go task with the electrical stimuli (Fig. 1). Electrical pulses were delivered at a rate of one per second for two minutes within each block. Subjects were told to respond with a button press to frequent single pulses (non-targets, 90\% of stimuli), but to withhold responses to infrequent double pulses (targets, $10 \%$ of stimuli, randomized).

During each task, 7/9 of the non-targets (single pulses) were 'low' intensity pulses. The remaining 2/9 pulses were distractors of a higher intensity delivered at random, which subjects were told to ignore; these were either non-painful ('medium' intensity) in condition 1, or painful ('high' intensity) in condition 2. Each condition was therefore equally matched for stimulus novelty, and differed only in the intensity of the pulses. In total, 2 blocks of each condition were performed, the order of which was counterbalanced (either 1-2-2-1 or 2-1-1-2, randomly assigned to each subject). After each block, subjects were asked to rate the average unpleasantness of the stimuli delivered in each block (including all painful and non-painful stimuli) using a 0-10 numerical scale ( $0=$ no sensation, $10=$ unbearable pain), which was anchored such that a level 4 indicated pain threshold.

\subsection{Experiment 2: Pain localization task. Measuring anticipatory ERPS to pain}

The high frequency of stimuli delivered during Experiment 1, and the co-incidence of motor-related processes occurring between stimuli, made it impossible to record a clean anticipatory-evoked potential that was specific to anticipating pain. In order to investigate potential neural correlates of the interruptive effect of pain anticipation on attention, and to differentiate different stages of pain anticipation as done previously (Brown et al., 2008), we designed a separate task in which pain stimuli were anticipated over a longer duration. Anticipatory ERP responses to pain were recorded as subjects focused attention on the spatial location of the anticipated painful stimuli. Subjects were therefore

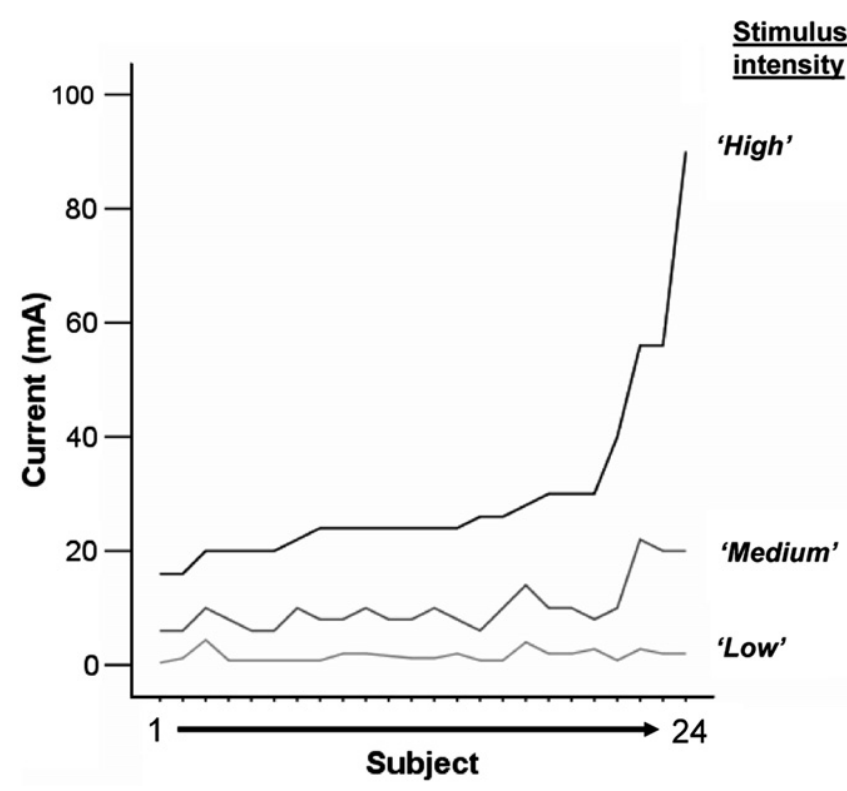

Fig. 2. The electrical current required to induce three intensity levels of pain stimulus for each subject The data is ordered according to the current needed to deliver a moderately painful ('high') intensity stimulus for each subject $(n=24)$. focused on the sensory-discriminative component of pain as during the go/no-go task, thus increasing the validity of making data comparisons between the two tasks. Laser stimuli were used during this task in order to identify responses specific to the anticipation of nociceptor activation (A $\delta$ - and C-fibre transmission), which lasers can achieve without the need for skin contact (Meyer et al., 1976).

Using a $\mathrm{CO}_{2}$ laser stimulator, heat stimuli of $150 \mathrm{~ms}$ duration and a beam diameter of $15 \mathrm{~mm}$ were applied to the dorsal surface of the subjects' right forearm. Subjects wore protective laser safety goggles during the experiment. Laser stimuli were randomly delivered to different positions on the arm over a skin area of $3 \times 5 \mathrm{~cm}$ in order to avoid habituation, sensitization or skin damage. As described previously (Bentley et al., 2004), this area was split into two adjacent halves of $1.5 \times 5 \mathrm{~cm}$ : a left half and a right half. During the experiment (details below) equal numbers of laser stimuli were delivered to each half in a pre-determined sequence.

An initial psychophysics procedure was performed using a 0-10 numerical scale, which was anchored such that a level 4 indicated just painful (pain threshold). A ramping procedure was repeated three times in order to determine a moderately painful level of laser stimulus intensity (level 7 on the scale) for each subject.

On each trial of the experiment (Fig. 3), a laser heat stimulus was delivered to the subject every $10 \mathrm{~s}$. Laser stimuli were preceded by the appearance of visual anticipation cues, displayed on a computer monitor in front of the subject, in order to provide subjects with accurate information about the timing of each laser stimulus. The anticipatory cue at $-3 \mathrm{~s}$ was a black dot; this changed to a gray dot at $-2 \mathrm{~s}$ and a white dot at $-1 \mathrm{~s}$. The white dot was displayed until $3 \mathrm{~s}$ after each laser stimulus, when a different visual cue was displayed in order to prompt the subjects to provide a response after each pulse relevant to the task.

Attending to the laser stimuli, subjects performed a pain localization task in two blocks of 15 trials. Subjects were asked to attend to the location of each laser stimulus during anticipation and experience of the pain, and decide whether they felt each pulse to be on the left or right half of the box on their arm. Subjects' peripheral vision was restricted using the laser safety goggles so that they were not able to visually perceive where each laser pulse had been delivered, and instead had to rely upon nociceptive sensation. Subjects responded to each laser pulse with one of the two possible button presses depending on whether they perceived a left- or right-sided pulse. After each block, subjects were asked to provide a rating of the average unpleasantness of the painful stimuli over the block using a 0-10 numerical scale.

In order to enable comparison of results between the go/no-go task and the pain localization task, the pain localization task took place after completing the first two blocks of go/no-go task, but before completing the final two blocks of the go/no-go task, resulting in a counterbalanced design. In doing so, the potential for confounds relating to differences in subjects' levels of tiredness between the two tasks was minimized.

\subsection{Electroencephalographic (EEG) recordings}

During the pain localization task, EEG recordings were taken from 61 scalp electrodes placed according to an extended 10-20 system (Quik-Cap system, Neuroscan, Inc.). Bandpass filters were set at DC $-100 \mathrm{~Hz}$, with a sampling rate of $500 \mathrm{~Hz}$ and gain of 500 . A notch filter was set to $50 \mathrm{~Hz}$ to reduce electrical interference. Electrodes were referenced to the ipsilateral (right) earlobe, and recordings were also taken from the contralateral (left) earlobe for off-line conversion to linked-ears reference. The vertical and horizontal electro-oculograms (EOG) were measured for off-line reduction of blink and eye-movement artifacts. 

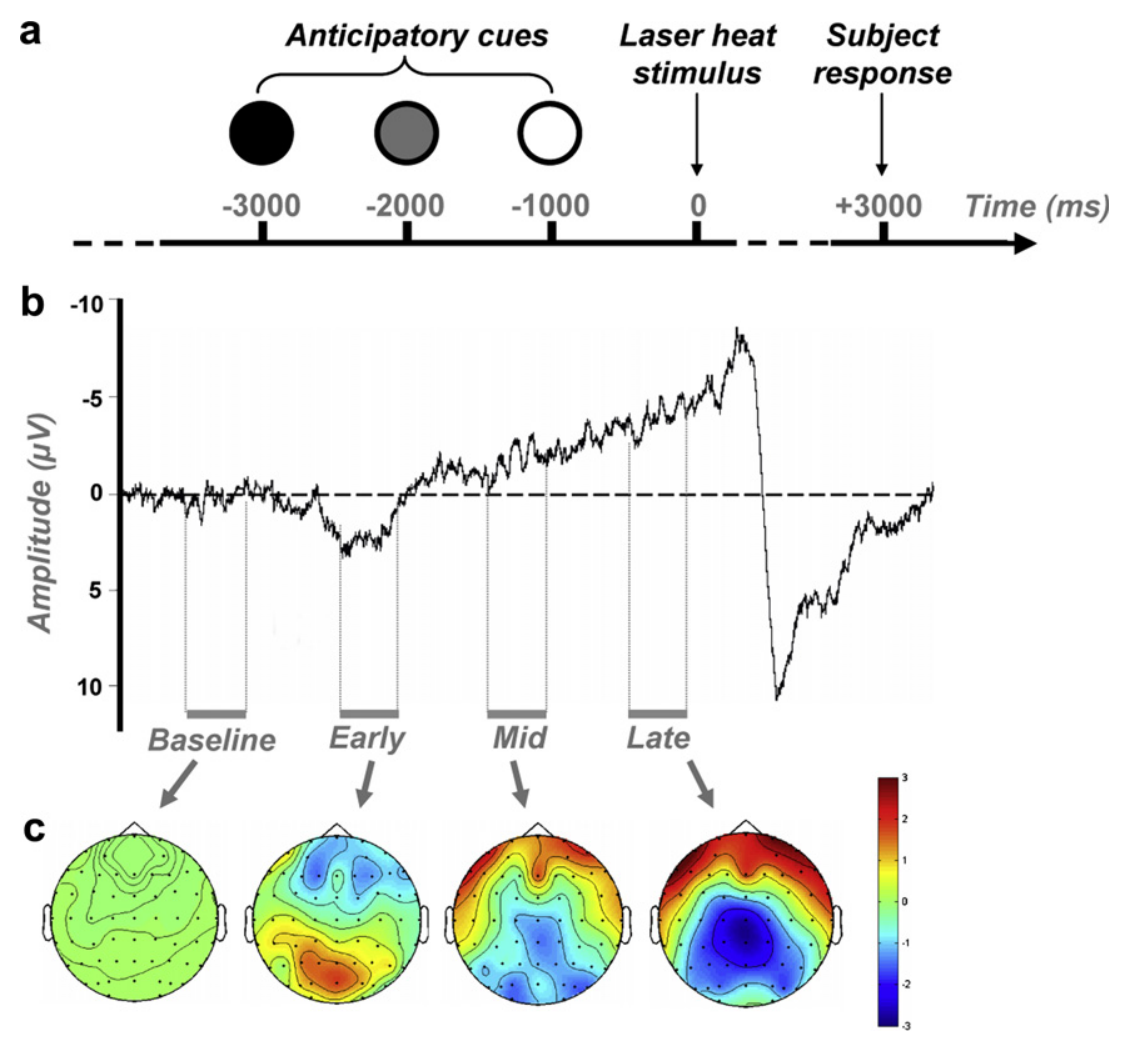

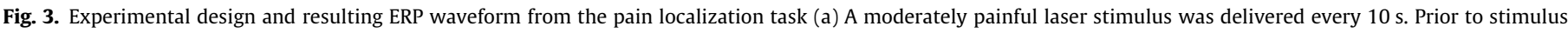

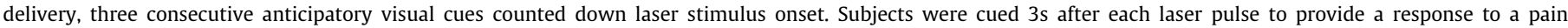

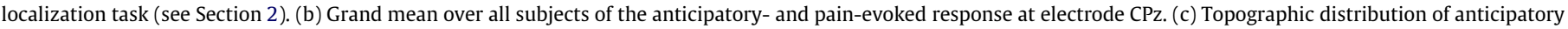
responses during baseline, early anticipation, mid-anticipation, and late-anticipation.

\subsection{Behavioral data analysis}

Task performance during the go/no-go task was determined from the number of errors made (i.e. subjects accidentally responding to targets), for each of the two conditions. We firstly used a Shapiro-Wilk test for normality to show that the data were not significantly different from the normal distribution, allowing us to use parametric statistics. We then determined whether there was a significant difference between the number of task errors occurring during condition 1 and condition 2 via paired-samples $t$-test. The relative increase in errors resulting from pain was calculated by subtracting the number of errors for condition 1 from the number of errors for condition 2 .

In order to determine whether increases in the perceived unpleasantness of the painful electrical stimuli (relative to the non-painful stimuli) were associated with increased task errors, post-task ratings of pain unpleasantness were averaged for each condition. Unpleasantness ratings for condition 1 were subtracted from those for condition 2 . The result was tested for normality using the Shapiro-Wilk test and then correlated with the increase in errors resulting from pain. The resulting relationship was tested using Pearson's correlation coefficient.

We also investigated whether subjects with a greater increase in error rates as a result of pain were more likely to judge any pain stimulus to be more unpleasant. To do this, we correlated the reported unpleasantness of the laser stimuli during the pain localization task with both the increase in unpleasantness of the electrical stimuli and the increase in error rates resulting from pain, using Pearson's correlation coefficient. It should be emphasized that the laser stimuli were delivered at an intensity that was determined independently of the electrical stimuli.
Next, we sought to discount the possibility that the electrical stimulus properties were determining changes in error rates rather than cognitive factors related to attention and perceived pain unpleasantness. Even though the psychophysics procedure was designed so that each subject would perceive the intensity of electrical stimuli to be the same, the applied current used for each subject may have been affected by bias in their conception of what constitutes pain threshold. This might lead to differences in the applied stimulus intensity that would affect error rates on the go/no-go task. We therefore investigated whether greater pain-related increases in error rates could be accounted for by a greater difference in the applied current between 'medium' and 'high' intensity stimuli. To do this, the increase in errors rates was correlated with the percentage increase in the delivered current, using Pearson's correlation coefficient. Similar analyses were performed to determine whether the percentage increase in delivered current of the electrical stimuli correlated with the percentage increase in pain unpleasantness ratings, and whether the delivered energy of the laser stimuli correlated with laser pain unpleasantness ratings.

\subsection{EEG data analysis}

EEG data from the pain localization task were analyzed using Neuroscan Edit 4.3 (Compumedics USA Ltd.). An ocular artifact reduction algorithm (Semlitsch et al., 1986) was performed. The data were epoched into single trials of $5.5 \mathrm{~s}$ duration starting $1000 \mathrm{~ms}$ before the first visual anticipation stimulus and ending $1500 \mathrm{~ms}$ seconds after the laser stimulus. Epochs were visually inspected for further ocular artifacts that had escaped automatic removal, and deleted if necessary. Linear trends over the whole epoch were removed using the entire epoch to calculate the linear 
component in all channels; the whole epoch was used in order to minimize removal of linear trends that may have specifically resulted from the anticipatory neural responses we were intending to measure. Each epoch was then baseline-corrected to the $500 \mathrm{~ms}$ interval preceding the visual anticipation cue. Trials were then averaged separately for each condition. Data were referenced to the common average before proceeding further with data analysis, although ERP waveforms are presented according to the linked-ears reference.

Three $500 \mathrm{~ms}$ phases of the anticipatory brain response were analyzed (Fig. 3). An 'early' phase, at $-2500 \mathrm{~ms}$ to $-2000 \mathrm{~ms}$ preceding the laser stimulus, was chosen as the earliest part of the anticipatory response that could be measured without interference from visual-evoked responses resulting from the first anticipation cue. A 'mid' phase was chosen at $-1500 \mathrm{~ms}$ to $-1000 \mathrm{~ms}$ preceding the laser stimulus. A 'late' phase, at $-500 \mathrm{~ms}$ to $0 \mathrm{~ms}$ preceding the laser stimulus, was chosen to represent processes taking place in immediate preparation for the impending laser stimulus.

Cortical sources of anticipatory ERPs at each time period were estimated with low-resolution electromagnetic tomography (LORETA), using the LORETA-KEY software (Pascual-Marqui et al., 2002). The software uses a three-shell spherical head model registered to the Talairach anatomical brain atlas (Talairach and Tournoux, 1988), although the electrode coordinates used were determined from a co-registration between spherical and realistic head geometry that create a best-fit model (Towle et al., 1993). LORETA estimates ERP sources in gray matter volume to a $7 \mathrm{~mm}^{3}$ grid resolution (2394 voxels in total) using the digitized MNI probability atlas (Mazziotta et al., 2001). Time-domain EEG files were converted to current density vector field magnitude using this technique. The resulting LORETA solutions were log-transformed at each pixel; this approximates LORETA solutions to a Gaussian distribution for parametric statistical analysis as previously demonstrated (Liu and Perfetti, 2003; Kiebel et al., 2005).

LORETA solutions were converted to SPM image format using a modified version of LOR2SPM (http://www.ihb.spb.ru/ pet_lab/ L2S/L2SMain.htm). During this process LORETA solutions were intensity-normalized in order to eliminate subject-to-subject global variations. Statistical maps were then created using SPM5 (http://www.fil.ion.ucl.ac.uk/spm/software/spm5) running on Matlab 7.1 (Mathworks Inc.). Initially, areas of the cortex activated during anticipation of pain were determined by performing a series of paired-samples $t$-tests for each anticipation period (early, mid, and late) relative to the baseline period. Results are reported at threshold of significance of $p<0.01$ (one-tailed, Family Wise Error (FWE) corrected for multiple comparisons), with a minimum of seven contiguous voxels.

Early, mid, and late phases of the anticipatory ERP data were analyzed separately as follows, using the sources identified as active during each of the three anticipation periods as masks to restrict results to these areas of interest. The relative change in error rates as a result of pain was entered as a covariate with LORETA sources during the pain localization task in order to test for correlations between changes in error rates (both increases and decreases) and current density at each voxel. Results are reported at an uncorrected threshold of significance of $p<0.05$ (two-tailed), with a minimum of seven contiguous voxels below this threshold, within our regions of interest (midcingulate and associated corti-
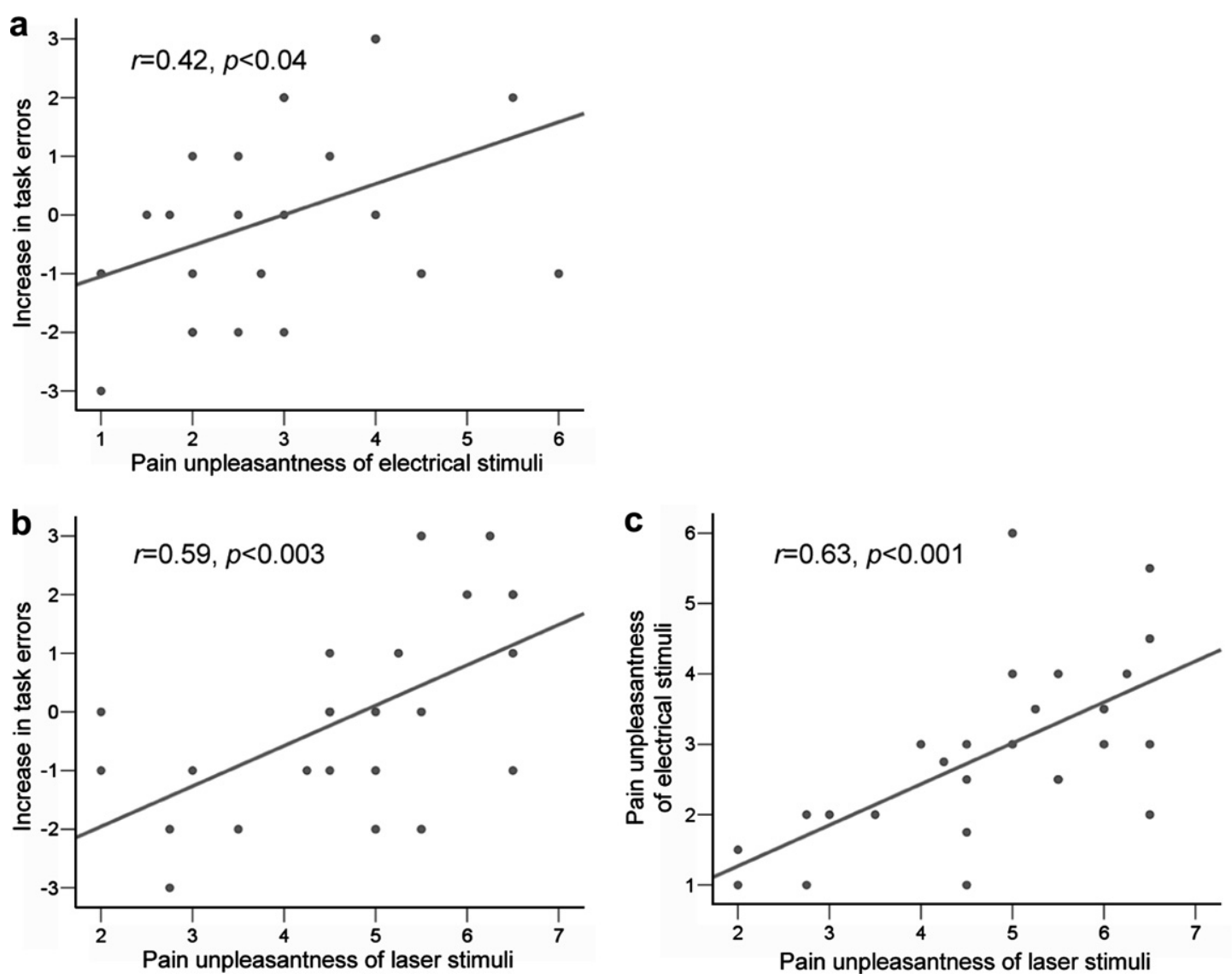

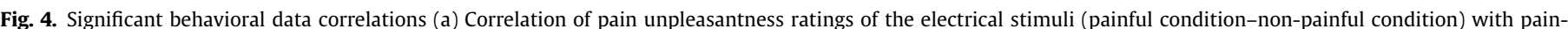

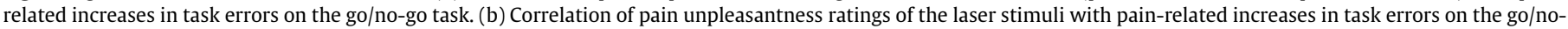

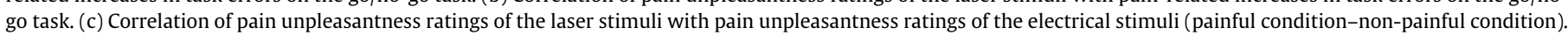


ces). Brain areas, revealed outside our areas of interest during this analysis, were only accepted at a corrected (FWE) threshold of significance of $p<0.05$ (two-tailed) with seven contiguous voxels.

Areas of the pain matrix that were above our statistical thresholds were further analyzed as volumes of interest (VOIs). VOIs were extracted as the first eigenvector (i.e. the mean of the adjusted response after rejecting noise) of all voxels within a sphere of $7 \mathrm{~mm}$ radius, centered on the most significantly activated voxel, that were above the chosen statistical threshold. These values are relative to a whole-brain mean signal of 100 . For each analysis described above, VOIs were correlated (using Pearson's coefficient) with the increase in unpleasantness ratings resulting from pain during the go/no-go task. We also correlated VOIs generated from the most significantly activated voxels within midcingulate cortex and inferior parietal cortex during each of the early- and late-anticipatory stages, in order to investigate differential co-variation of these loci at the two time points.

\section{Results}

In comparing the relative number of errors occurring during the go/no-go task, we found that there was no significant difference between the painful and non-painful conditions across all subjects. The mean $( \pm \mathrm{SD})$ number of errors was $4.38( \pm 5.2)$ for the painful task and $4.58( \pm 5.0)$ for the non-painful task. However, the relative increase in the perceived unpleasantness of the electrical stimuli (Experiment 1), after introducing randomized painful stimuli, correlated with pain-related increases in errors on that task $(r=0.42$, $p<0.04$; Fig. 4). Furthermore, the reported unpleasantness of the
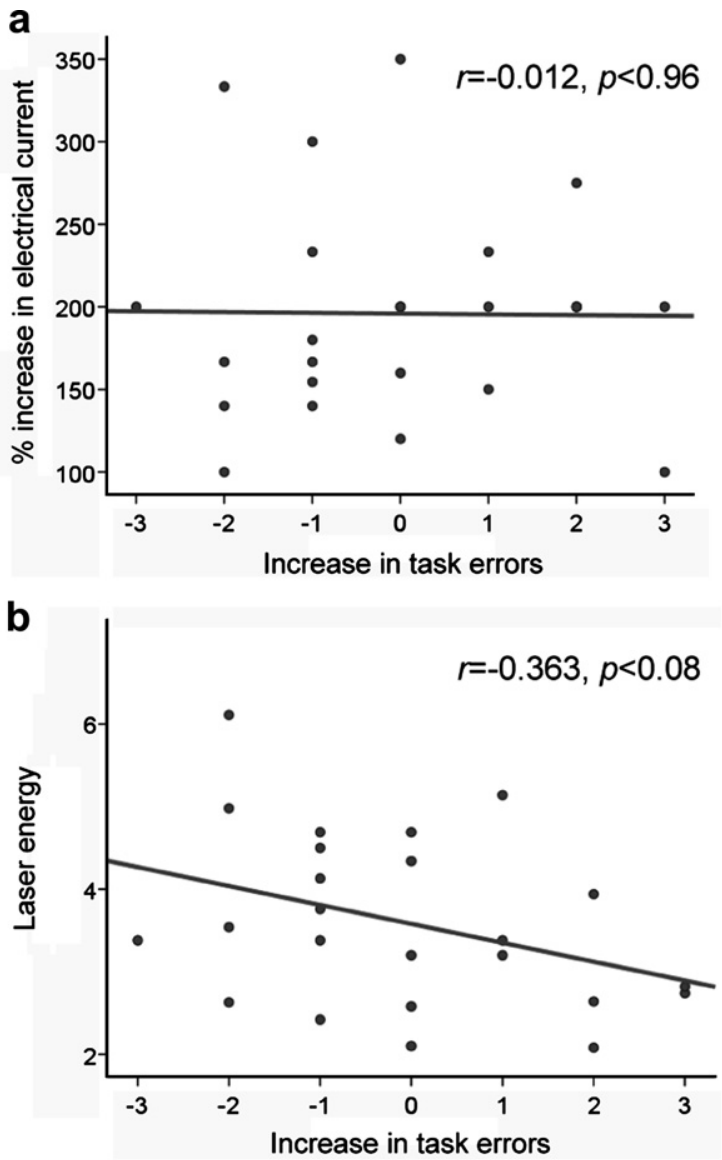

laser stimuli during the pain localization task (Experiment 2) correlated with two behavioral measures from the go/no-go task (Experiment 1): pain-related increases in the unpleasantness of the electrical stimuli $(r=0.63, p<0.001)$, and the pain-related increases in error rates $(r=0.59, p<0.003)$. Interestingly, there was no correlation between the percentage increase in the delivered electrical current of the stimuli and either the increase in error rates or the increase in perceived unpleasantness in Experiment 1 (Fig. 5). There was also no correlation between the delivered laser energy during the pain localization task and unpleasantness ratings during Experiment 2.

The EEG results show differential activation during the three anticipatory stages in the pain matrix (Table 1, Fig. 6). Pain matrix areas activated during early anticipation include bilateral posterior insula, midcingulate cortex, posterior cingulate cortex, bilateral inferior parietal lobe, and right hippocampus, in addition to bilateral precentral gyri and areas of the middle and superior temporal lobe. During mid-anticipation, activation was more restricted with increases in current density only found in bilateral posterior insula and parahippocampal gyrus. Late-anticipation was characterized by similar activity in posterior insula and hippocampal areas, with additional activation of midcingulate, posterior cingulate, precentral gyrus, and superior temporal lobe. Unlike during early anticipation, mid, and late anticipation did not reveal activation of inferior parietal lobe.

Increased error rates as a result of introducing painful distractors in the go/no-go task (Experiment 1) were associated with greater anticipatory activity in midcingulate cortex as subjects attended to the location of anticipated painful laser stimuli (Experi-

\section{C}
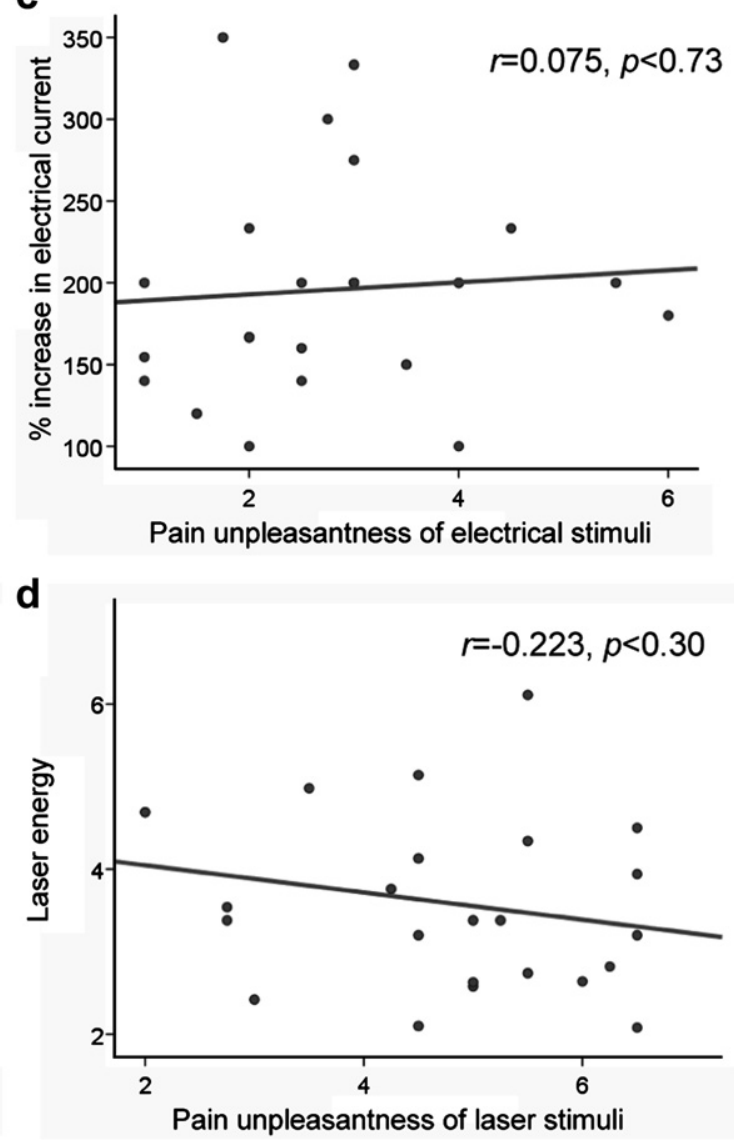

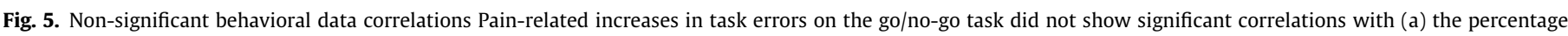

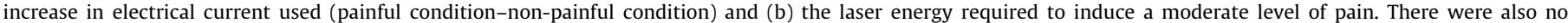

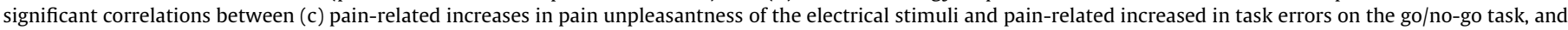
(d) pain unpleasantness ratings of the laser stimuli and the laser energy required to induce a moderate level of pain. 
Table 1

Brain regions showing increased current density during anticipation of pain during three separate temporal stages

\begin{tabular}{|c|c|c|c|c|c|c|c|}
\hline \multirow[t]{2}{*}{ Brain region } & & \multirow[t]{2}{*}{ Area } & \multicolumn{3}{|c|}{ MNI coordinates } & \multirow[t]{2}{*}{$t$-Value } & \multirow[t]{2}{*}{$p$-Value } \\
\hline & & & $x$ & $y$ & $z$ & & \\
\hline \multicolumn{8}{|l|}{ Early anticipation } \\
\hline \multirow[t]{2}{*}{ Posterior insula } & $\mathrm{R}$ & 13 & 39 & -18 & 22 & 12.24 & 0.000 \\
\hline & $\mathrm{L}$ & 13 & -31 & -18 & 1 & 11.11 & 0.000 \\
\hline \multirow[t]{2}{*}{ Precentral gyrus } & $\mathrm{R}$ & 6 & 46 & -11 & 36 & 8.16 & 0.000 \\
\hline & $\mathrm{L}$ & 43 & -52 & -11 & 15 & 9.29 & 0.000 \\
\hline \multirow[t]{2}{*}{ Inferior parietal lobule } & $\mathrm{R}$ & 40 & 39 & -32 & 43 & 7.77 & 0.000 \\
\hline & $\mathrm{L}$ & 40 & -31 & -39 & 43 & 7.58 & 0.000 \\
\hline Superior temporal gyrus & $\mathrm{R}$ & 13 & 46 & -46 & 22 & 6.39 & 0.000 \\
\hline Hippocampus & $\mathrm{R}$ & - & 25 & -39 & 1 & 10.94 & 0.000 \\
\hline Middle temporal gyrus & $\mathrm{L}$ & 39 & -45 & -53 & 15 & 8.16 & 0.000 \\
\hline Middle occipital gyrus & $\mathrm{L}$ & 18 & -24 & -95 & 15 & 7.75 & 0.000 \\
\hline Midcingulate cortex & $\mathrm{L}$ & 24 & -3 & -4 & 29 & 10.91 & 0.000 \\
\hline Precuneus & $\mathrm{R}$ & 39 & 25 & -60 & 22 & 10.43 & 0.000 \\
\hline Posterior cingulate cortex & $\mathrm{R}$ & 29 & 4 & -46 & 8 & 8.74 & 0.000 \\
\hline \multicolumn{8}{|l|}{ Mid-anticipation } \\
\hline \multirow[t]{2}{*}{ Parahippocampal gyrus } & $\mathrm{R}$ & 30 & 11 & -39 & 1 & 13.05 & 0.000 \\
\hline & $\mathrm{L}$ & 30 & -17 & -39 & 1 & 10.78 & 0.000 \\
\hline \multirow[t]{2}{*}{ Posterior insula } & $\mathrm{R}$ & 13 & 39 & -18 & 1 & 8.06 & 0.000 \\
\hline & $\mathrm{L}$ & 13 & -31 & -11 & 1 & 6.85 & 0.000 \\
\hline \multicolumn{8}{|l|}{ Late-anticipation } \\
\hline Hippocampus & $\mathrm{R}$ & - & 25 & -39 & 1 & 10.13 & 0.000 \\
\hline Parahippocampal gyrus & $\mathrm{R}$ & 30 & 32 & -53 & 1 & 8.53 & 0.000 \\
\hline Lingual gyrus & $\mathrm{L}$ & 19 & -24 & -67 & -6 & 6.52 & 0.000 \\
\hline Midcingulate cortex & $\mathrm{L}$ & 24 & -3 & -4 & 29 & 6.82 & 0.000 \\
\hline Posterior cingulate cortex & $\mathrm{L}$ & 29 & -3 & -39 & 22 & 5.82 & 0.000 \\
\hline \multirow[t]{2}{*}{ Posterior insula } & $\mathrm{R}$ & 13 & 39 & -18 & 22 & 9.52 & 0.000 \\
\hline & $\mathrm{L}$ & 13 & -31 & -11 & 1 & 9.39 & 0.000 \\
\hline Precentral gyrus & $\mathrm{L}$ & 43 & -52 & -11 & 15 & 7.26 & 0.000 \\
\hline Superior temporal gyrus & $\mathrm{R}$ & 42 & 67 & -25 & 15 & 6.46 & 0.000 \\
\hline
\end{tabular}

Corr, corrected p-values (FWE); area, Brodmann's area.

ment 2) during early anticipation only (Fig. 6). Increased errors were also associated with reduced current density during early anticipation in the right inferior parietal cortex and posterior cingulate cortex (Fig. 6). There was no correlation of increased errors with current density during mid or late-anticipation anywhere within the areas activated during pain anticipation. Furthermore, no correlations were found between pain-related increases in unpleasantness ratings on the go/no-go task and current density in midcingulate cortex, right inferior parietal cortex or posterior cingulate cortex.

Highly significant correlations of current density were found between midcingulate and right inferior parietal cortices during late anticipation $(r=0.70, p<0.001$; Fig. $7 b)$. However, during early anticipation correlations between the two regions did not reach significance despite a trend toward a positive correlation $(r=0.39, p<0.06$; Fig. 7a).

\section{Discussion}

Our first hypothesis is supported by the behavioral results: task-irrelevant painful stimuli during the go/no-go task increased error rates more in subjects reporting the greatest increases in pain unpleasantness (Fig. 4). There was no significant difference between the painful and non-painful conditions of the task over the whole group, suggesting that pain only has an interruptive effect on attention in a subgroup of individuals, i.e. those who find it to be the most unpleasant. Furthermore, subjects with the greatest pain-related increases in task errors were generally biased toward reporting the emotional component of pain regardless of the stimulus properties. Despite the fact that the electrical and laser stimuli used in the two tasks were tailored independently of each other to produce a pre-specified subjective intensity level, subjects who reported greater unpleasantness in response to the painful electrical stimuli also reported the laser stimuli to be more unpleasant. Importantly, the delivered electrical current of the painful electrical stimuli did not correlate with either the perceived unpleasantness of the stimuli nor with the increased task errors they caused. The delivered energy of the laser pulses also did not correlate with the unpleasantness ratings of these stimuli (Fig. 5). This suggests that subjects with increased pain-related task errors were biased in their reporting of pain toward emphasizing its emotional characteristics, regardless of the sensory characteristics of the stimulus. The variation in pain unpleasantness ratings is therefore best explained by differences in attention to the emotional characteristics of the pain rather than by its stimulus intensity.

\subsection{Neural activity during anticipation of pain}

The results showing widespread neural activation over the pain matrix (Fig. 6a) during anticipation of pain is consistent with fMRI studies showing similar areas of activation during anticipation and experience of acute pain (Hsieh et al., 1999; Ploghaus et al., 1999; Porro et al., 2002). With the finer temporal resolution of EEG, we were able to show that the most widespread activation of the pain matrix was during the early-anticipatory stage. More specifically, early anticipation was associated with co-activation of the midand posterior cingulate cortices with bilateral inferior parietal cortices, which did not take place during mid- and late-anticipatory stages. Right inferior parietal and midcingulate cortices are heavily interconnected anatomically (Baleydier and Mauguiere, 1980; Vogt and Pandya, 1987), and substantial evidence implicates these regions in attention, particularly with respect to pain (Coull and Nobre, 1998; Posner and Rothbart, 1998; Peyron et al., 1999; Sawamoto et al., 2000; Behrmann et al., 2004; Buffington et al., 2005; Fan et al., 2005).

More restricted areas of the pain matrix (bilateral posterior insula and parahippocampal gyrus) were activated during mid-anticipation, with a notable lack of cingulate and parietal cortex activation. Hippocampal areas are involved with maintaining memory traces that enable associative learning in aversive conditioning (Buchel et al., 1999). Although LORETA source solutions are less robust for deeper brain sources (Trujillo-Barreto et al., 2004), research suggests strong agreement between LORETA sources and intra-cerebral recordings in deep mesial temporal/hippocampal areas (Zumsteg et al., 2005).

Previous data have shown that late, but not early, anticipatory processing predicts the amplitude of the pain-evoked response when the intensity of the forthcoming pain is predictable (Brown et al., 2008). In this study, late-anticipatory processing was associated with cingulate activity in the absence of inferior parietal cortical activity. However, there were high correlations of current density between midcingulate and inferior parietal areas during late anticipation, in contrast to the poor correlation during early anticipation. Spatial correlations can be used to imply the degree of functional connectivity between two regions, or can otherwise indicate common connections to a third (or more) neuronal dri$\operatorname{ver}(\mathrm{s})$. We speculate therefore that attention areas (midcingulate and inferior parietal cortices) may function within a more cohesive network during late stages of anticipation, potentially involving further neuronal systems not detectable using surface EEG such as the thalamus. It has been previously suggested that such changes in connectivity may facilitate top-down influences on pain (Tracey, 2005).

\subsection{Anticipatory processes influencing attention and pain unpleasantness}

Further EEG results (Fig. 6b) support our second hypothesis, that anticipatory responses to pain in midcingulate cortex are asso- 
a

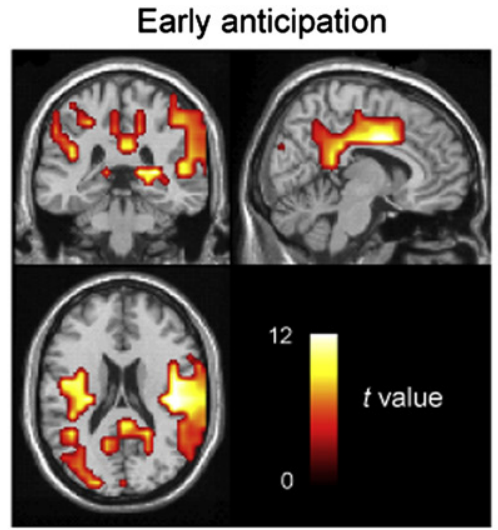

Mid anticipation

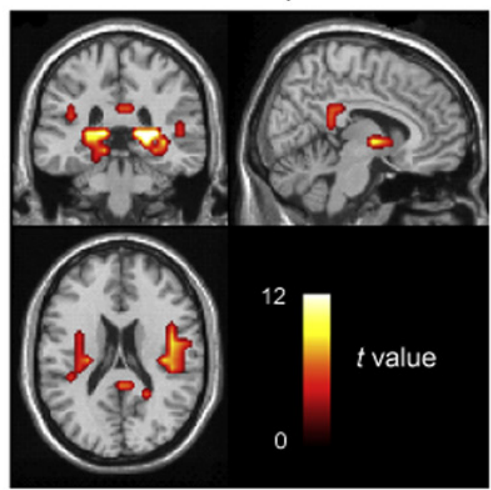

Late anticipation

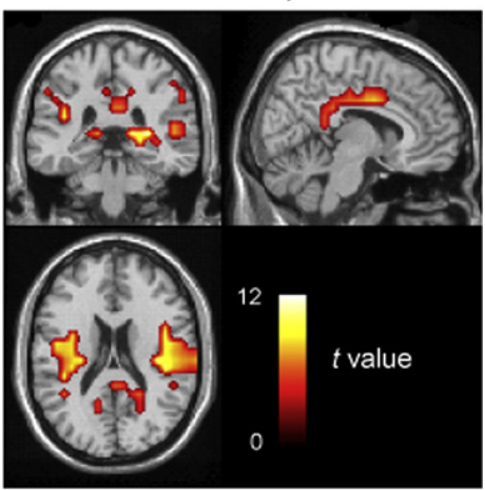

b
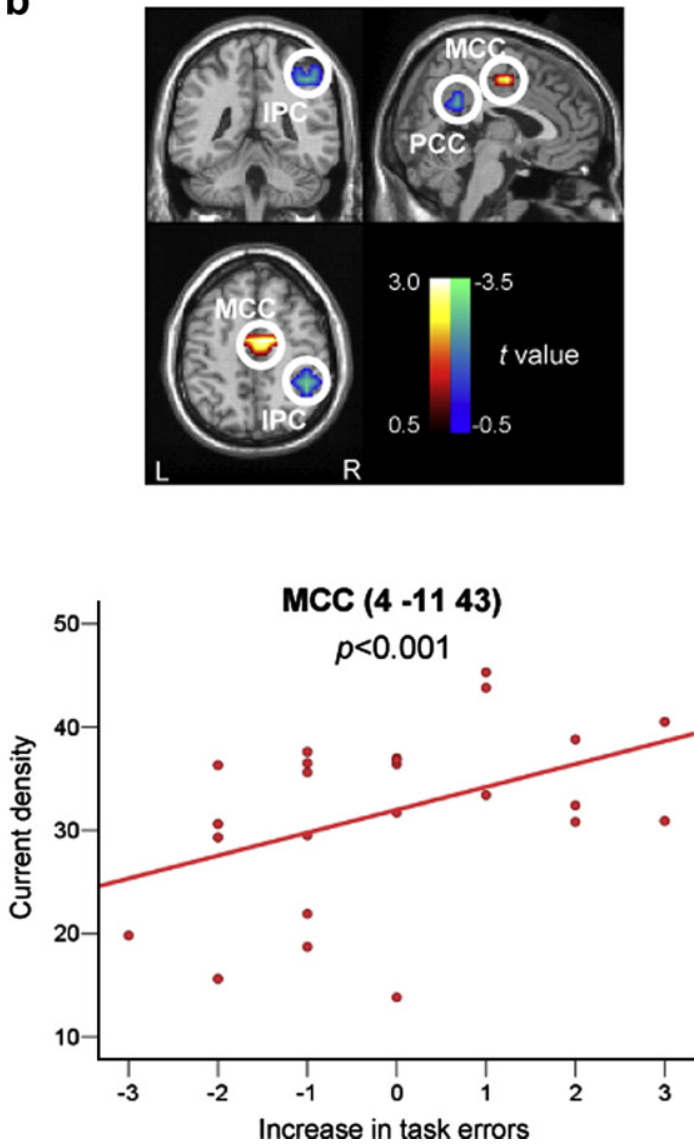
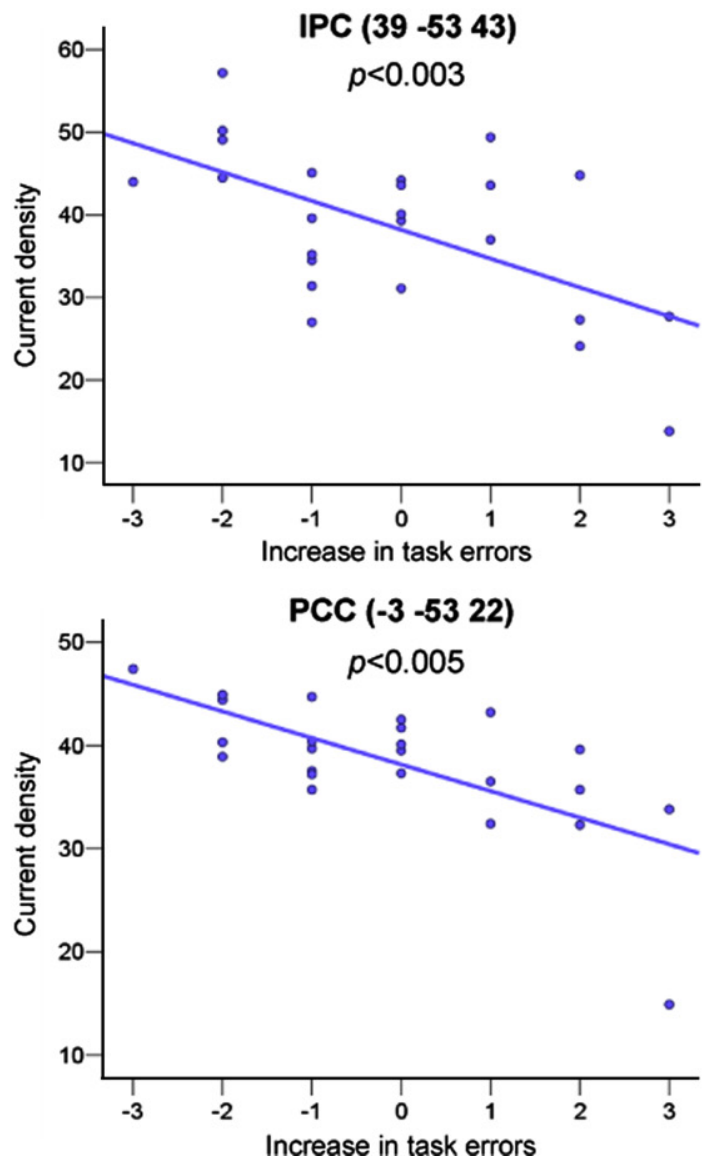

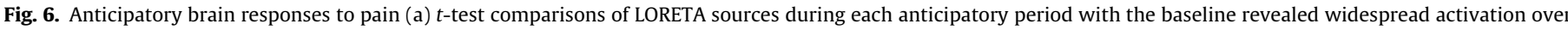

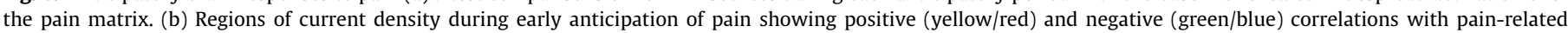
increases in task errors on the go/no-go task (MCC: midcingulate cortex; PCC: posterior cingulate cortex; IPC: inferior parietal cortex).

ciated with greater attentional interruption by pain anticipation. Subjects with the greatest pain-related increases in task errors during the go/no-go task showed greater activations of midcingulate cortex during the early-anticipatory stage. Recent research using fMRI implicates attention networks involving anterior midcingulate cortex in the interruptive effects of pain (Seminowicz and Davis, 2007). The more posterior region of the midcingulate located in this study is consistent with the known differences between fMRI and EEG in estimating sources of brain activity related to pain processing (Garcia-Larrea et al., 2003).

Pain-related increases in task errors were associated with reduced anticipatory activation of right inferior parietal cortex and posterior cingulate cortex. These areas are interconnected (Vogt,
2005) and known to form part of a network involved with spatial orientation of attention (Peyron et al., 1999; Behrmann et al. 2004; Kulkarni et al., 2005; Fan et al., 2005). The poor correlation between midcingulate and inferior parietal attention areas during early anticipation is reflected in the dissociable relationship of these regions to errors in the go/no-go task.

Together, our EEG results support the hypothesis that midcingulate responses during anticipation of pain are associated with attentional interruption. However, it is unclear from the present results what mechanisms mediate this relationship. A model that has been previously described (e.g. Peyron et al., 2000) suggests that midcingulate areas direct attention toward pain automatically in response to a stimulus, and are therefore reflective of 'bottom- 

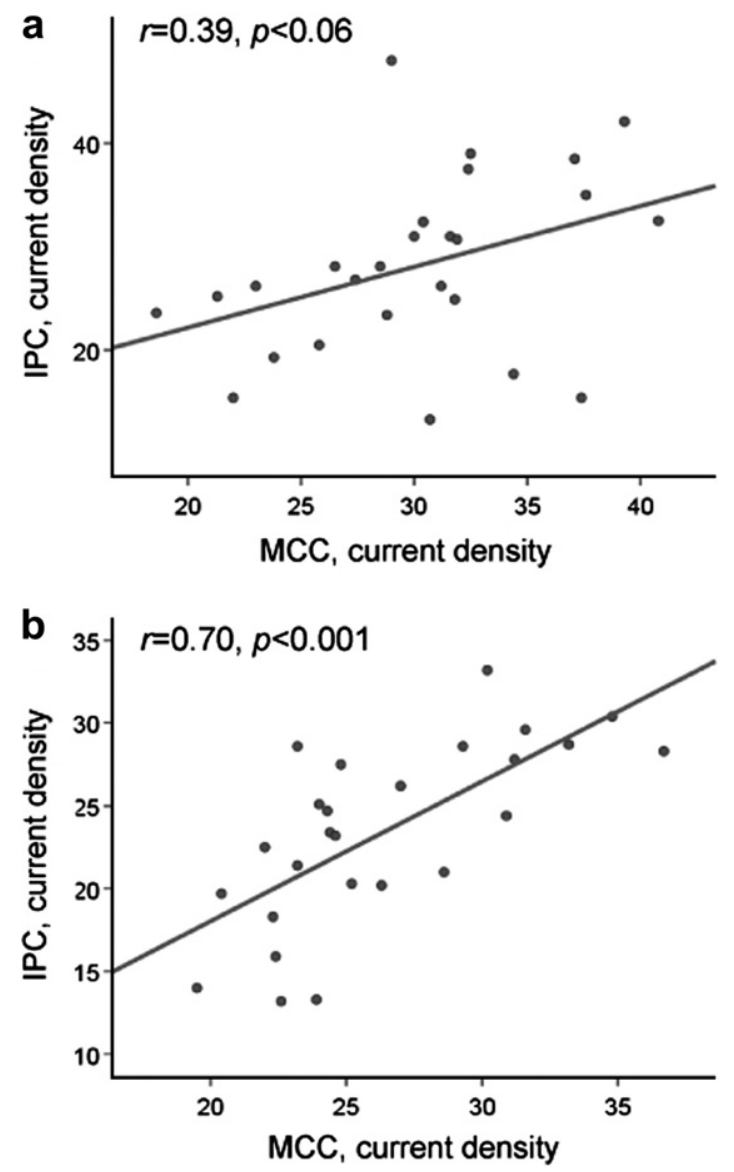

Fig. 7. Correlated activity in attention areas during anticipation (a) Correlations between current density in midcingulate cortex (MCC; MNI coordinates: $-3,-4$, 29) and right inferior parietal cortex (IPC; MNI coordinates: 39, -32, 43) during early anticipation of pain did not reach statistical significance. However, during late-anticipation (b) the same regions were found to be highly correlated.

up' (sensory-driven) processes. This is consistent with our finding that early-anticipatory midcingulate responses (just post-anticipation cue), rather then late responses (just prior to pain), are associated with attentional interruption. Hypervigilance toward cues that predict pain may be one possible driver of attentional interruption. However, we have not established whether greater early midcingulate responses result from true 'bottom-up' processing or whether there may be some involvement of subcortical areas such as the amygdala that mediate fast-conditioned responses to fear-conditioned anticipation stimuli. This possibility is also suggested by our behavioral data which show that attentional interruption is related to the factors that influence the emotional response to pain.

The EEG results also indicate that during late-anticipation midcingulate areas become more highly connected to inferior parietal areas, which are known to be involved in directing attention toward pain (Peyron et al., 1999). An intriguing question is to what extent early-anticipatory midcingulate responses (which may determine attentional interruption by pain) have an influence over the subsequent connectivity of attention networks as the pain stimulus approaches. A more detailed analysis using effective connectivity models would be required to answer this question.

\subsection{Limitations of the study}

One of the aims of the study was to determine the anticipatory neural correlates of attentional interruption, using the modified go/no-go task as a measure of sustained attention. Even though target stimuli and the subsequent non-target stimuli were never painful, this does not exclude the possibility that a painful stimulus may have led to prolonged disruptions of attention or motor preparatory responses needed for the task that may have also increased errors. Hence, it is not absolutely guaranteed that the attentional interruption effects resulting from painful stimuli in Experiment 1 can be solely attributed to anticipatory effects. However, this limitation would only serve to increase the potential for false-negative results when correlating pain-related errors with anticipatory neural activity. Therefore, we are confident that our results are valid within the degree of error afforded by the statistical thresholds employed.

A further noteworthy limitation arises from our collection of retrospective ratings of pain unpleasantness after the end of each task, which lasted about two minutes. Although pain ratings are always likely to be influenced by memory biases, whether given immediately after a painful stimulus or minutes after, this factor may be more important with increased time. For example, previous research has suggested that retrospective ratings of chronic pain become distorted over a period of weeks (Linton and Melin, 1982, 1998). On a shorter time scale that is comparable to that used in this study, other research has shown that placebo analgesic effects are more pronounced when judged based on retrospective pain ratings compared to concurrent ratings (Price et al., 1999). It is unclear how memory may have affected the data in this study, but again we would only expect an increased potential for falsenegative results when correlating retrospective ratings with the more immediate effects of pain anticipation, i.e. its influence on task performance.

The causality of the relationship between the perception of pain unpleasantness, increased pain-related errors on the go/no-go task, and increased anticipatory midcingulate responses cannot be firmly established with the present data. However, as discussed above, there is some evidence from the results that those subjects with increased errors in the go/no-go task as a result of pain may have been biased toward attending to the emotional component of pain, suggesting that cognitive factors were causative in establishing the relationship between task performance and pain unpleasantness.

Moreover, although our results show that anticipatory midcingulate responses are associated with increased pain-related task errors, midcingulate responses may not have been causally related to the increased errors. One complication within the experimental design was that we correlated results from two separate tasks, making inferences about causality all the more difficult. Further work could test the importance of midcingulate responses in determining attentional functions in relation to pain by using transcranial magnetic stimulation to produce temporary and reversible lesions to this area of the brain.

\subsection{Clinical implications}

Disability in patients with chronic pain is strongly influenced by anticipatory fear, which is more predictive of disability than the actual intensity of the pain (Leeuw et al., 2007). However, disability may also be related to attentional dysfunction in such patients (Dick et al., 2002). A number of studies have demonstrated interruptive effects of pain or its anticipation on attentional performance, showing that attentional interruption is increased by fear (Crombez et al., 1998; Crombez et al., 1998; Eccleston and Crombez, 1999; Boston and Sharpe, 2005). There is evidence that fear of pain is associated with stronger pain-related brain activity in the midcingulate cortex (Ochsner et al., 2006), which may act to direct attention toward the pain, consistent with our data. 
Our results have implications for the development of surrogate biomarkers of attentional dysfunction in chronic pain. Some psychological therapies for chronic pain are geared toward the use of attentional training as a pain management strategy (McCracken, 2005). Patients with greater anticipatory midcingulate responses may be predisposed to hypervigilance toward clinical pain symptoms and be more likely to benefit from attentional training.

\section{Acknowledgements}

This work was funded by the University of Manchester and the Arthritis Research Campaign. Thanks to Professor Steve Williams and Dr. Lloyd Gregory at the University of Manchester for their support of this research.

\section{References}

Arntz A, Dreessen L, De Jong P. The influence of anxiety on pain: attentional and attributional mediators. Pain 1994;56:307-14.

Baleydier C, Mauguiere F. The duality of the cingulate gyrus in monkey. Neuroanatomical study and functional hypothesis. Brain 1980;103:525-54.

Behrmann M, Geng JJ, Shomstein S. Parietal cortex and attention. Curr Opin Neurobiol 2004;14:212-7.

Bentley DE, Watson A, Treede RD, Barrett G, Youell PD, Kulkarni B, et al. Differential effects on the laser evoked potential of selectively attending to pain localisation versus pain unpleasantness. Clin Neurophysiol 2004;115:1846-56.

Bocker KB, Brunia CH, Van den Berg-Lenssen MM. A spatiotemporal dipole model of the stimulus preceding negativity (SPN) prior to feedback stimuli. Brain Topogr 1994;7:71-88.

Bogels SM, Mansell W. Attention processes in the maintenance and treatment of social phobia: hypervigilance, avoidance and self-focused attention. Clin Psychol Rev 2004;24:827-56.

Boston A, Sharpe L. The role of threat-expectancy in acute pain: effects on attentional bias, coping strategy effectiveness and response to pain. Pain 2005;119:168-75.

Brown CA, Seymour B, Boyle Y, El-Deredy W, Jones AKP. Modulation of pain perception by expectation and uncertainty: behavioral characteristics and anticipatory neural correlates. Pain 2008;135:240-50.

Brunia CH, van Boxtel GJ. Wait and see. Int J Psychophysiol 2001;43:59-75.

Buchel C, Dolan RJ, Armony JL, Friston KJ. Amygdala-hippocampal involvement in human aversive trace conditioning revealed through event-related functional magnetic resonance imaging. J Neurosci 1999;19:10869-76.

Buffington AL, Hanlon CA, McKeown MJ. Acute and persistent pain modulation of attention-related anterior cingulate fMRI activations. Pain 2005;113:172-84.

Bush G, Luu P, Posner MI. Cognitive and emotional influences in anterior cingulate cortex. Trends Cogn Sci 2000;4:215-22.

Coull JT, Nobre AC. Where and when to pay attention: the neural systems for directing attention to spatial locations and to time intervals as revealed by both PET and fMRI. J Neurosci 1998;18:7426-35.

Crombez G, Eccleston C, Baeyens F, Eelen P. Attentional disruption is enhanced by the threat of pain. Behav Res Ther 1998;36:195-204.

Crombez G, Eccleston C, Baeyens F, Eelen P. When somatic information threatens, catastrophic thinking enhances attentional interference. Pain 1998;75:187-98.

Crombez G, Eccleston C, Baeyens F, Van Houdenhove B, Van den BA. Attention to chronic pain is dependent upon pain-related fear. J Psychosom Res 1999;47:403-10.

Crombez G, Van Damme S, Eccleston C. Hypervigilance to pain: an experimental and clinical analysis. Pain 2005;116:4-7.

Davis KD, Taylor SJ, Crawley AP, Wood ML, Mikulis DJ. Functional MRI of pain- and attention-related activations in the human cingulate cortex. J Neurophysiol 1997;77:3370-80

Derbyshire SW, Vogt BA, Jones AK. Pain and Stroop interference tasks activate separate processing modules in anterior cingulate cortex. Exp Brain Res 1998;118:52-60.

Dick B, Eccleston C, Crombez G. Attentional functioning in fibromyalgia, rheumatoid arthritis, and musculoskeletal pain patients. Arthritis Rheum. Arthritis Care Res. 2002;47:639-44.

Eccleston C, Crombez G. Pain demands attention: a cognitive-affective model of the interruptive function of pain. Psychol Bull 1999;125:356-66.

Fan J, McCandliss BD, Fossella J, Flombaum JI, Posner MI. The activation of attentional networks. NeuroImage 2005;26:471-9.

Feine JS, Lavigne GJ, Dao TTT, Morin C, Lund JP. Memories of chronic pain and perceptions of relief. Pain 1998;77:137-41.

Garcia-Larrea L, Frot M, Valeriani M. Brain generators of laser-evoked potentials: from dipoles to functional significance. Neurophysiol Clin 2003;33:279-92.
Hsieh JC, Stone-Elander S, Ingvar M. Anticipatory coping of pain expressed in the human anterior cingulate cortex: a positron emission tomography study. Neurosci Lett 1999;262:61-4.

Keogh E, Ellery D, Hunt C, Hannent I. Selective attentional bias for pain-related stimuli amongst pain fearful individuals. Pain 2001;91:91-100.

Kiebel SJ, Tallon-Baudry C, Friston KJ. Parametric analysis of oscillatory activity as measured with EEG/MEG. Hum Brain Mapp 2005;26:170-7.

Kulkarni B, Bentley DE, Elliott R, Youell P, Watson A, Derbyshire SW, et al. Attention to pain localization and unpleasantness discriminates the functions of the medial and lateral pain systems. Eur J Neurosci 2005;21:3133-42.

Leeuw M, Goossens ME, Linton SJ, Crombez G, Boersma K, Vlaeyen JW. The fearavoidance model of musculoskeletal pain: current state of scientific evidence. J Behav Med 2007;30:77-94.

Linton SJ, Melin L. The accuracy of remembering chronic pain. Pain 1982;13:281-5.

Liu Y, Perfetti CA. The time course of brain activity in reading English and Chinese: an ERP study of Chinese bilinguals. Hum Brain Mapp 2003;18:167-75.

Mazziotta J, Toga A, Evans A, Fox P, Lancaster J, Zilles K, et al. A probabilistic atlas and reference system for the human brain: International Consortium for Brain Mapping (ICBM). Philos Trans R Soc Lond B Biol Sci 2001;356:1293-322.

McCracken LM. Contextual cognitive behavioral therapy for chronic pain. IASP Press; 2005.

Meyer RA, Walker RE, Mountcastle Jr VB. A laser stimulator for the study of cutaneous thermal and pain sensations. IEEE Trans Biomed Eng 1976;23:54-60.

Nutt DJ, Ballenger JC, Sheehan D, Wittchen HU. Generalized anxiety disorder: comorbidity, comparative biology and treatment. Int J Neuropsychopharmacol 2002;5:315-25.

Ochsner KN, Ludlow DH, Knierim K, Hanelin J, Ramachandran T, Glover GC, et al. Neural correlates of individual differences in pain-related fear and anxiety. Pain 2006;120:69-77.

Pascual-Marqui RD, Esslen M, Kochi K, Lehmann D. Functional imaging with lowresolution brain electromagnetic tomography (LORETA): a review. Methods Find Exp Clin Pharmacol 2002;24(Suppl. C):91-5.

Peters ML, Vlaeyen JW, van Drunen C. Do fibromyalgia patients display hypervigilance for innocuous somatosensory stimuli? Application of a body scanning reaction time paradigm. Pain 2000;86:283-92.

Peyron R, Garcia-Larrea L, Gregoire MC, Costes N, Convers P, Lavenne F, et al. Haemodynamic brain responses to acute pain in humans: sensory and attentional networks. Brain 1999;122(Pt 9):1765-80.

Peyron R, Laurent B, Garcia-Larrea L. Functional imaging of brain responses to pain A review and meta-analysis. Neurophysiol Clin 2000;30:263-88.

Ploghaus A, Tracey I, Gati JS, Clare S, Menon RS, Matthews PM, et al. Dissociating pain from its anticipation in the human brain. Science 1999;284:1979-81.

Porro CA, Baraldi P, Pagnoni G, Serafini M, Facchin P, Maieron M, et al. Does anticipation of pain affect cortical nociceptive systems? J Neurosci 2002;22:3206-14.

Posner MI, Petersen SE. The attention system of the human brain. Annu Rev Neurosci 1990;13:25-42.

Posner MI, Rothbart MK. Attention, self-regulation and consciousness. Philos Trans R Soc Lond B Biol Sci 1998;353:1915-27.

Price DD, Milling LS, Kirsch I, Duff A, Montgomery GH, Nicholls SS. An analysis of factors that contribute to the magnitude of placebo analgesia in an experimental paradigm. Pain 1999;83:147-56.

Sawamoto N, Honda M, Okada T, Hanakawa T, Kanda M, Fukuyama H, et al. Expectation of pain enhances responses to nonpainful somatosensory stimulation in the anterior cingulate cortex and parietal operculum/posterior insula: an event-related functional magnetic resonance imaging study. J Neurosci 2000;20:7438-45.

Seminowicz DA, Davis KD. Pain enhances functional connectivity of a brain network evoked by performance of a cognitive task. J Neurophysiol 2007;97:3651-9.

Seminowicz DA, Mikulis DJ, Davis KD. Cognitive modulation of pain-related brain responses depends on behavioral strategy. Pain 2004;112:48-58.

Semlitsch HV, Anderer P, Schuster P, Presslich O. A solution for reliable and valid reduction of ocular artifacts, applied to the P300 ERP. Psychophysiology 1986;23:695-703.

Talairach J, Tournoux P. Co-planar stereotaxic atlas of the human brain. New York: Thieme; 1988.

Towle VL, Bolanos J, Suarez D, Tan K, Grzeszczuk R, Levin DN, et al. The spatial location of EEG electrodes: locating the best-fitting sphere relative to cortical anatomy. Electroencephalogr Clin Neurophysiol 1993;86:1-6.

Tracey I. Functional connectivity and pain: how effectively connected is your brain? Pain 2005;116:173-4.

Trujillo-Barreto NJ, Aubert V, Valdés-Sosa PA. Bayesian model averaging in EEG MEG imaging. NeuroImage 2004;21:1300-19.

Vogt BA. Pain and emotion interactions in subregions of the cingulate gyrus. Nat Rev Neurosci 2005;6:533-44.

Vogt BA, Pandya DN. Cingulate cortex of the rhesus monkey: II. Cortical afferents. J Comp Neurol 1987;262:271-89.

Zumsteg D, Friedman A, Wennberg RA, Wieser HG. Source localization of mesial temporal interictal epileptiform discharges: correlation with intracranial foramen ovale electrode recordings. Clin Neurophysiol 2005;116:2810-8. 